\title{
Validation of a Novel Trial Oral Appliance Protocol Versus a Conventional Custom Oral Appliance Protocol for the Treatment of Obstructive Sleep Apnea
}

Daniel J Levendowski, MBA ${ }^{1}$; Edward Sall, MD, DDS²; Todd Morgan, DMD³; Bretton Beine, RPSGT4; William Odom, DDS 3 ; Dorian Cruz Arista, DA ${ }^{3}$; Dominic Munafo, MD ${ }^{4}$

${ }^{1}$ Advanced Brain Monitoring, Inc., Carlsbad, CA, USA; ${ }^{2}$ BetterNight Sleep, Inc, San Diego, CA, USA; ${ }^{3}$ Sleep Alliance LLC, San Diego, CA, USA; ${ }^{4}$ Sleep Data, Inc., San Diego, CA, USA.

\begin{abstract}
Study Objective: The goal of the study is to compare the treatment response achieved with a trial oral appliance (OA) to that obtained with a custom OA.

Methods: A within-subject, repeated-measures, case-control design was performed in which 193 patients were fitted with an Apnea Guard $®$ trial OA (AG), with the vertical dimension of occlusion (VDO) dependent on sex and tongue scallop and protruded to a target setting. Two-night sleep studies assessed response to the AG (i.e., night $1=$ baseline, night $2=\mathrm{AG}$ ). Eighty-four responders to the AG were subsequently assessed with a custom OA (CA), fabricated and titrated according to a conventional protocol. Comparisons were then made between the CA and AG.

Results: Both males and females showed significant improvements in the overall and supine apnea-hypopnea index (AHI) values, independent of $\mathrm{OA}$ and tongue scallop (all $P<0.01$ ). Relative to the $\mathrm{CA}$, the AG provided significantly greater reductions in the overall AHI values $(P<0.002)$. Both OAs significantly reduced the nonsupine AHIs in males, but not in females. The proportion of subjects who achieved an AHI $<10$ events/h was $44.0 \%$ with the $\mathrm{CA}$ versus $73.8 \%$ with the AG $(P<0.0001)$. The percentages of those who achieved $\geq 50 \%$ AHI reductions with the $\mathrm{CA}$ and $\mathrm{AG}$ were $52.4 \%$ and $77.4 \%$, respectively $(P<0.002)$. Applying logistic regression to posttreatment AHIs obtained from the two OAs across three outcome criteria, VDO (odds ratio: 1.34-1.83, all $P<0.006$ ) and pretreatment overall AHI values (odds ratio: 0.88-1.05, all $P<0.02$ ) consistently predicted therapeutic response.
\end{abstract}

Conclusion: The results from this study demonstrated that the AG protocol, which selectively accommodates sex and tongue scallop, provided treatment responses superior to a conventional CA protocol.

Keywords: mandibular advancement, obstructive sleep apnea, oral appliance, treatment outcome, tongue scallop, vertical dimension of occlusion

Citation: Levendowski DJ, Sall E, Morgan T, et al. Validation of a novel trial oral appliance protocol versus a conventional custom oral appliance protocol for the treatment of obstructive sleep apnea. J Dent Sleep Med. 2021;8(2)

\section{INTRODUCTION}

In most developed countries of the world, oral appliance therapy (OAT) is the second most recommended treatment for obstructive sleep apnea (OSA). ${ }^{1,2}$ It is, however, prescribed less than one-tenth as often as positive airway pressure (PAP). ${ }^{3-5}$ OAT is most often used in patients intolerant of PAP, despite the fact that OAT is often preferred over PAP, and by some measures is as effective as PAP (after consideration of both efficacy and compliance) ${ }^{6,7}$ OAT is not commonly used as a first-line therapy for several reasons. First, a custom, titratable oral appliance (OA) is more expensive than PAP, in part, because of the time and effort required to fabricate, fit, and optimize the appliance. ${ }^{8,9}$ Second, the proportion of patients who achieve a good outcome with an oral appliance range from $50 \%$ to $70 \%$, depending on the endpoint criteria. ${ }^{6,10-13}$ But unlike with PAP, patients cannot currently undergo a relatively inexpensive trial to determine whether they will respond to and/or tolerate an OA. As a result, the healthcare systems in many countries pay for PAP, but not OAT.

Journal of Dental Sleep Medicine
A number of investigations have attempted to characterize clinical phenotypes for use as selection criteria to increase the odds of a successful OA outcome. Both sex and positional OSA have been reported to influence the success of OA therapy. ${ }^{10,14-16}$ Polysomnographic factors associated with greater OA efficacy included lower loop gain, higher arousal threshold, lower response to arousals, moderate pharyngeal collapsibility, and weaker muscle compensation. ${ }^{17}$ Cone beam computed tomography was used to assess the effects of tongue scallop, adipose tissue, pharyngeal length, hyoid position, ethnicity, and sex facial phenotypes on upper airway collapse. ${ }^{6,18-22}$ Cephalometric features were able to distinguish OA success in a group of Taiwanese patients (area under the receiver operating characteristic curve, 86\%), however, these findings could not be repeated in an Australian cohort. ${ }^{13,23}$ Using sleep endoscopy, patients with a posteriorly located tongue exhibited a more robust response to OA therapy. ${ }^{24}$ Determination of OA treatment response has also been investigated using a remotely controlled mandibular protrusion device, initially evaluated in a laboratory setting 
and later during multinight in-home recordings. The resulting dichotomous responder/no-responder decision was initially based on $\geq 50 \%$ AHI reduction combined with an $\mathrm{AHI}<10$ events/h, and later based solely on an $\mathrm{AHI}<10$ events/h. ${ }^{11,12,25}$ Despite these efforts, a simple, inexpensive option that allows clinicians and patients to readily identify the degree of response that can be expected with OAT has yet to emerge.

The primary objective of this study was to compare the reductions in sleep-disordered breathing achieved with the Apnea Guard ${ }^{\circledR}(\mathrm{AG})$ trial OA, using a protocol that selects functional settings based on sex and tongue scallop and protrudes to a "target" setting, versus a custom OA (CA) fitted, fabricated, and titrated according to conventional dental protocol. Upon completion, post hoc analyses were performed to identify patient characteristics and/or OA functional settings associated with differences in $\mathrm{AG}$ and $\mathrm{CA}$ outcomes.

\section{METHODS}

\section{Data Acquisition}

A within-subject, repeated-measures, case-control study design was conducted prospectively in a clinical setting to evaluate adult patients referred with a diagnostic AHI $\geq 5$ events/h and who were intolerant of PAP therapy. All patients had appropriate dentition for OAT (i.e., at least eight teeth in the upper and lower arch).

In addition to the initial diagnostic study, a 2-night assessment home sleep apnea test (HSAT) (NightOne, Philips Healthcare, Monroeville PA) was performed at baseline: on night 1 without an OA and on night 2 with the AG (described in the next paragraphs). A 1-night efficacy HSAT was ordered after the CA titration had been completed. Apneas were detected based on $\geq 90 \%$ reduction in airflow for 10 seconds, and hypopneas required $\geq 30 \%$ change in airflow coupled with a $3 \%$ oxyhemoglobin desaturation. HSAT studies were reviewed by one of two board certified sleep medicine physicians.

Two hundred thirteen patients were fitted with the AG between May 2018 and October 2019 (Figure 1). Of these, 193 completed the assessment HSAT and 20 patients did not (16 cases unrelated to the AG). In total, $72 \%$ (138) responded to the AG based on criteria developed for a costcontainment program during the 1 -year period prior to the start of this study. These criteria required either $\geq 40 \%$ reduction in the overall $\mathrm{AHI}$ when the pretreatment $(\mathrm{PT})$ AHI severity was $\geq 20$ or $\geq 30 \%$ reduction with a PT AHI severity $<20$ events $/ \mathrm{h}$. These criteria were designed to limit mischaracterization of OA responders that could result in denial of care due to (1) night-to-night variability in milder OSA; (2) the increased incidence of mild OSA using a 3\% rather than a $4 \%$ desaturation criteria ${ }^{26}$; and (3) the potential inferiority of the $A G$ relative to a $C A$. The descriptive data for the 84 cases who completed the efficacy HSAT are presented in Table 1. There were no significant differences in descriptive data between those fitted with the CA and who did versus who did not complete the efficacy HSAT.

Table 1. Descriptive data for patients who completed custom oral appliance efficacy home sleep apnea tests

\begin{tabular}{l|l}
\hline$*=$ Mean $+/-\mathrm{SD}$ & $\mathrm{n}=84$ \\
\hline Males, $\mathrm{n}(\%)$ & $46(54.8)$ \\
Age, years * & $53.8 \pm 11.9$ \\
BMI kg/m ${ }^{2} *$ & $29.4 \pm 5.7$ \\
Neck size, cm * & $39.7 \pm 4.5$ \\
Overall AHI, events/h $*$ & $24.6 \pm 14.4$ \\
Supine AHI, events/h $*$ & $32.9 \pm 18.0$ \\
Nonsupine AHI, events/h $*$ & $12.4 \pm 11.1$ \\
Mild OSA, $\mathrm{n}(\%)$ & $20(23.8)$ \\
Moderate OSA, $\mathrm{n}(\%)$ & $44(52.4)$ \\
Severe OSA, $\mathrm{n}(\%)$ & $20(23.8)$
\end{tabular}

$\mathrm{AHI}$, apnea-hypopnea index; BMI, body mass index; OSA, obstructive sleep apnea.

\section{Oral Appliances}

The AG (Advanced Brain Monitoring, Carlsbad, CA) was selected with the distance between the upper and lower teeth imposed by the AG with the jaw closed and stationary (i.e., vertical dimension of occlusion (VDO)) based on sex and tongue scallop. Males with nonscalloped tongues were fitted with an AG size "medium" (i.e., $6.5 \mathrm{~mm}$ anterior and $5.5 \mathrm{~mm}$ posterior interocclusal distance) and males with scalloped tongues were delivered an AG size "high" (i.e., $8.0 \mathrm{~mm}$ anterior and $6.5 \mathrm{~mm}$ posterior interocclusal distance). Females with nonscalloped tongues were fitted with an AG size "low" (i.e., $5.5 \mathrm{~mm}$ anterior and posterior interocclusal distance) and those with scalloped tongues were fitted with an AG size "medium". ${ }^{27}$

Tongue scallop was determined using a technique very similar to obtaining a Mallampati score. Instead of patients protruding their tongue as far as possible, they were instructed to rest the tip of the relaxed tongue on the bottom edge of the lower lip and drop the mouth open. The degree of tooth markings on the lateral borders of the tongue were then observed (Figure 2). A higher VDO was selected in cases with marginal scalloping. Both the dentists and dental assistants applied this AG selection technique over a 1-year period and in more than 75 patients prior to the start of this study.

The "target" protrusion was set to $70 \%$ of the distance from centric occlusion to maximum protrusion with the AG in situ. The accuracy of this method in identifying the optimal jaw-forward position was previously described. ${ }^{28}$ The AGs were fitted by a dental assistant, typically in less than 15 minutes. The CAs were fabricated according to 


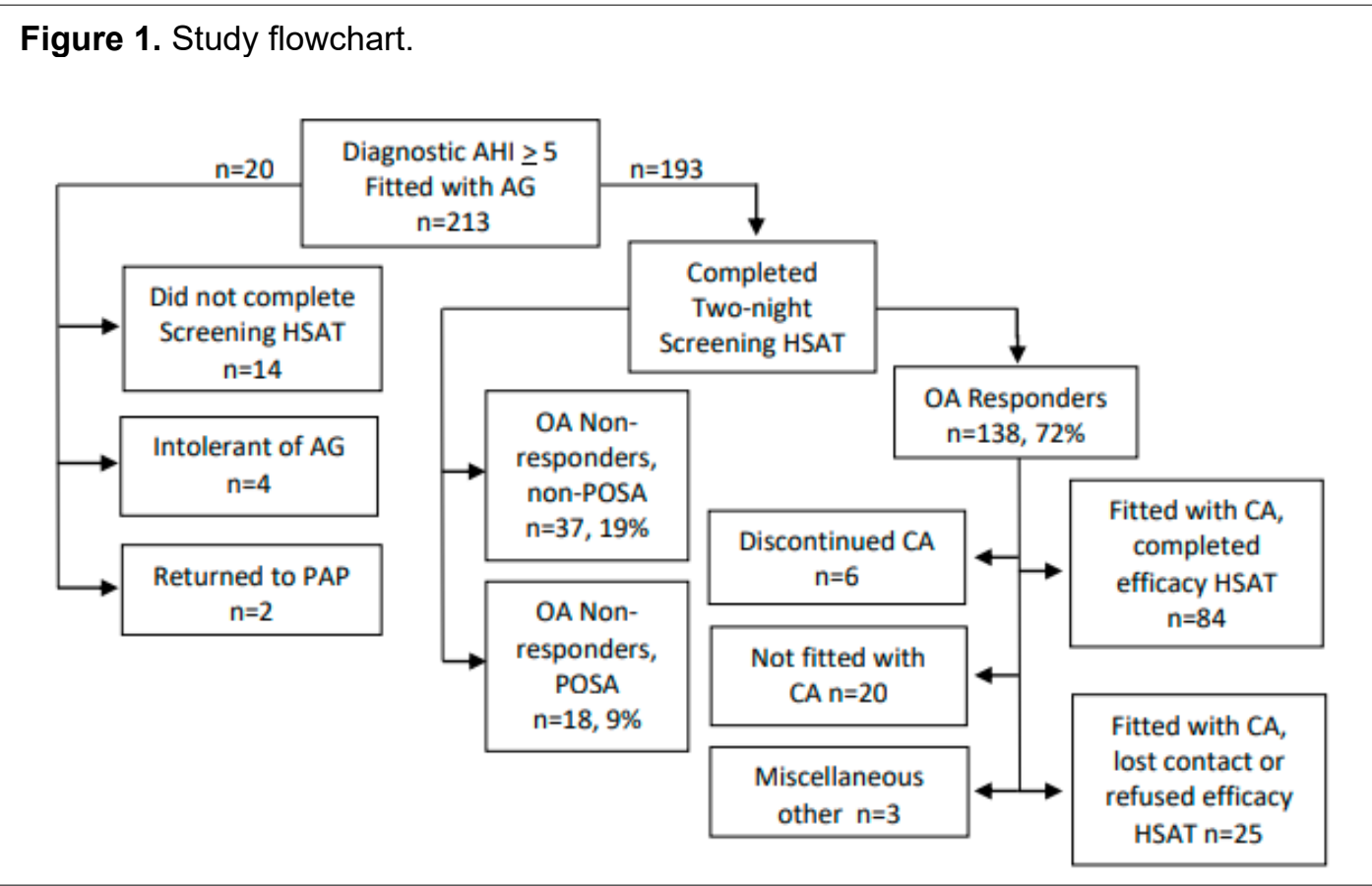

Figure 2. Images of a nonscalloped tongue (a), scalloped tongue (b), and AG trays for three VDO sizes (c).

a.

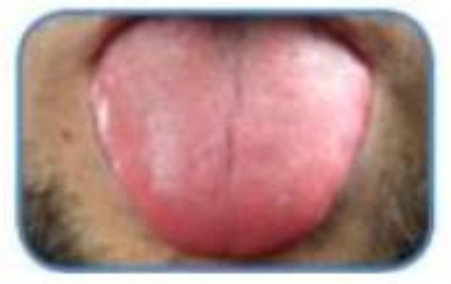

b.

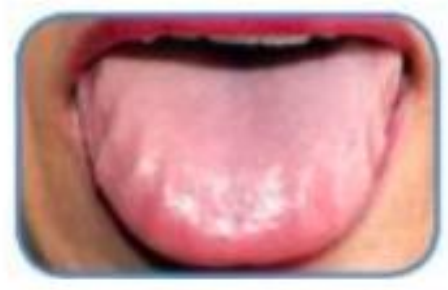

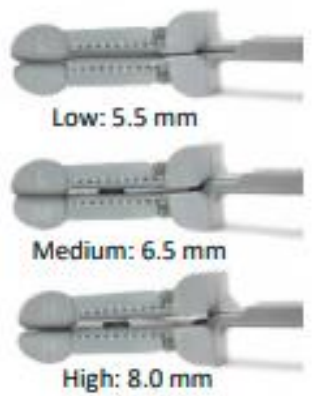

conventional dental protocols. Initial protrusion was set to $70 \%$ of range of motion from maximum retrusion to maximum protrusion using a George Gauge bite fork. Females were prescribed a CA with an approximately 2$\mathrm{mm}$ VDO (i.e., the actual VDO ranged from 2 to $3 \mathrm{~mm}$ depending on the fabrication limitations of the CA) and males were fitted with a 5-mm VDO. The CAs included $51 \%$ Herbst (True Function Lab, La Mesa, CA or Great Lakes Orthodontics, Tonawanda, NY), 25\% D-SAD (Panthera Dental, Quebec Canada), 14\% $\mathrm{MicrO}_{2}{ }^{\circledR}$ (ProSomnus ${ }^{\circledR}$ Sleep Technologies, Pleasanton, CA) and $10 \%$ other. All patients were instructed on how to apply the vertical elastics to the CA (used to secure the upper and lower trays) if they experienced dry mouth or snoring (resulting from the jaw falling open during the night). The CA was titrated with protrusion advanced until the dentist determined the patient had achieved a successful outcome based on reported resolution of snoring, somnolence, and other nocturnal and/or daytime symptoms, similar to the protocol used by de Ruiter et al. ${ }^{29}$

\section{Data Analysis}

Patients' records were accessed post hoc with approval from the BioMed Institutional Review Board (San Diego, CA). PT AHI values were selected from either the diagnostic or the baseline study, whichever was greater. Patients were categorized as having mild, moderate, or severe OSA based on standard AHI criteria. A minimum of 20 minutes of recording time was required to compute supine and nonsupine AHI values. Positional OSA (POSA) was characterized when the overall AHI divided by the nonsupine AHI was $\geq 1.4$ events $/ \mathrm{h} .{ }^{26}$

Mann-Whitney $U$ tests were used to compare group differences with significant findings reported after Bonferroni corrections. Logistic regression models based on age, sex, neck size, body mass index (BMI), tongue scallop, PT AHI, and the VDO were used to identify predictors of posttreatment outcomes. The CA logistic regression also included OA design type (i.e., Herbst, D$\mathrm{SAD}$, MicrO2, Other), but excluded VDO because it was 
dichotomously equivalent to sex. The AG logistic regression excluded tongue scallop, given its selection was reliant on sex and VDO. Logistic regression outcomes were assessed using three criteria: a posttreatment overall $\mathrm{AHI}<10$ events $/ \mathrm{h} ; \geq 50 \%$ reduction from the overall pretreatment $\mathrm{AHI}$; and $\geq 50 \% \mathrm{AHI}$ reduction in combination with an $\mathrm{AHI}<10$ events/h.

\section{RESULTS}

\section{Primary Findings: Changes in AHI by OA}

Both the $\mathrm{CA}$ and the $\mathrm{AG}$ significantly reduced the overall, supine, and nonsupine AHI values (all $P<0.002$; Figure 3). Relative to the $\mathrm{CA}$, the $\mathrm{AG}$ provided significantly greater reductions in the overall AHIs. The percentage of patients who achieved an $\mathrm{AHI}<10$ events $/ \mathrm{h}$ was $44.0 \%$ with the $\mathrm{CA}$ versus $73.8 \%$ with the $\mathrm{AG}$ $(P<0.0001)$; the percentage of patients who achieved $\geq 50 \%$ AHI reductions was $52.4 \%$ and $77.4 \%$, respectively $(P<0.002)$. The percentage of patients who achieved $\geq 50 \%$ AHI reduction in combination with an $\mathrm{AHI}<10$ events $/ \mathrm{h}$ was $32.1 \%$ with the CA vs. $59.5 \%$ with the AG $(P<0.001)$.

Figure 3. Pretreatment, $C A$, and $A G$ overall, supine and nonsupine $\mathrm{AHI}$ values (mean \pm standard error).

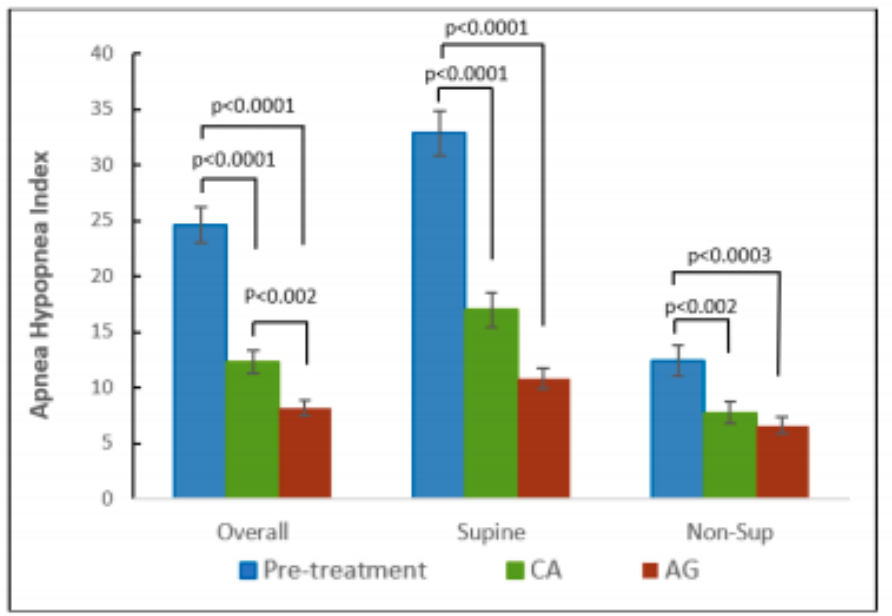

\section{Secondary Findings: Pretreatment Differences by Sex and Tongue Scallop}

Significant differences between males and females were apparent across all descriptive and AHI severity measures (Table 2). In this cohort of patients intolerant of PAP, males had significantly larger neck circumferences and greater pretreatment overall, supine, and nonsupine AHI values compared to females. Females had slightly higher BMI values and a greater percentage had mild OSA. The distributions of nonscalloped and scalloped tongues in males versus females were equivalent. Males and females with scalloped tongues had higher nonsupine AHI values and a greater percentage had severe OSA in comparison with their respective counterparts with nonscalloped tongues. A significantly greater percentage of males with nonscalloped tongues had POSA in comparison with those who had scalloped tongues.

\section{Secondary Findings: Changes in AHI by OA, Sex, Tongue Scallop, and Position}

Both males and females exhibited significant reductions overall and supine AHI values, independent of OA and tongue scallop (Table 3). Both OAs provided significant reductions in nonsupine AHI values in males, independent of tongue scallop. In males with scalloped tongues, the AG delivered significantly greater reductions in overall AHIs, as compared with the $\mathrm{CA}(P<0.01)$.

\section{Secondary Findings: Predictors of Outcomes Across and by Sex and OA}

Tables 4 and 5 present logistic regression variables that were significant for at least one condition $(P<0.05)$. Across sexes and OAs, the variables sex, PT AHI, and VDO predicted OA success across the three outcome criteria. BMI was predictive in the two outcome criteria that required $\mathrm{a} \geq 50 \% \mathrm{AHI}$ reduction.

When stratified by sex, additional predictive variables emerged. Tongue scallop and VDO were predictive in males across all three outcome criteria, but not for females. In females, BMI was predictive in those achieving $\geq 50 \%$ $\mathrm{AHI}$ reduction in combination with an $\mathrm{AHI}<10$ events $/ \mathrm{h}$, whereas VDO was only predictive for the outcome criteria $\mathrm{AHI}<10$ events $/ \mathrm{h}$. PT AHI was a significant predictor of males and females who achieved a posttreatment $\mathrm{AHI}<10$ events $/ \mathrm{h}$, in males with $\geq 50 \mathrm{AHI}$ reduction, and in females with a $\geq 50 \%$ AHI reduction in combination with an $\mathrm{AHI}<10$ events/h.

PT AHI was the only CA predictor for two of the outcome criteria, and no variable predicted a CA outcome based on a $\geq 50 \%$ AHI reduction in combination with an $\mathrm{AHI}<10$ events/h (notwithstanding inclusion of OA design type; Table 5). For the AG logistic regression, BMI and PT AHI predicted outcomes across all three criteria, whereas VDO was predictive for the two outcome criteria requiring $\geq 50$ AHI reduction. In an alternative regression model, tongue scallop provided similar predictive capabilities when substituted for VDO.

\section{DISCUSSION}

The results from this study demonstrated that the AG, selectively fitted for males and females, was effective in 
Table 2. Descriptive data stratified by sex and tongue scallop

\begin{tabular}{|c|c|c|c|c|c|c|c|c|c|}
\hline & \multicolumn{3}{|c|}{ All } & \multicolumn{3}{|c|}{ Males: by tongue scallop } & \multicolumn{3}{|c|}{ Females: by tongue scallop } \\
\hline & Males & Females & $P$ & $\begin{array}{c}\text { Non- } \\
\text { scalloped }\end{array}$ & Scalloped & $P$ & $\begin{array}{c}\text { Non- } \\
\text { scalloped }\end{array}$ & Scalloped & $P$ \\
\hline Subjects, n (\%) & $46(54.8)$ & $38(45.2)$ & --- & $16(34.8)$ & $30(65.2)$ & --- & $14(36.8)$ & $24(63.2)$ & --- \\
\hline Age, year* & $51.8 \pm 11.2$ & $56.2 \pm 12.4$ & 0.09 & $49.8 \pm 12.2$ & $52.9 \pm 10.7$ & 0.379 & $58.2 \pm 9.2$ & $55.0 \pm 14.0$ & 0.484 \\
\hline BMI $\mathrm{kg} / \mathrm{m}^{2} *$ & $29 . \overline{1}+4.2$ & $29 . \overline{6}+7.2$ & $<0.0001$ & $28.0+4.0$ & $29 . \overline{8}+4.3$ & 0.174 & $26.1+5.1$ & $31 . \overline{7}+7.4$ & 0.023 \\
\hline Neck Size, $\mathrm{cm} *$ & $42.4+3.3$ & $36.4+3.5$ & $<0.0001$ & $41.5+2.9$ & $42.9+3.5$ & 0.242 & $35.4+3.2$ & $37.0+3.5$ & 0.227 \\
\hline Overall AHI, events/h* & $28.0 \pm 14.6$ & $20.5 \pm 13.1$ & 0.004 & $22.1 \pm 6.1$ & $31.2 \pm 16.8$ & 0.136 & $16.3 \pm 6.9$ & $22.9 \pm 15.2$ & 0.363 \\
\hline Supine AHI, events/h * & $38.4 \pm 19.5$ & $26.6 \pm 13.8$ & 0.002 & $31.4 \pm 11.2$ & $42.7 \pm 22.3$ & 0.089 & $25.9 \pm 9.7$ & $27.0 \pm 16.0$ & 0.653 \\
\hline Non-Sup AHI, events/h * & $15.2 \pm 12.2$ & $9.1 \pm 8.5$ & 0.015 & $8.5 \pm 4.2$ & $19.1 \pm 13.6$ & 0.007 & $5.0 \pm 5.4$ & $11.6 \pm 9.2$ & 0.018 \\
\hline Mild OSA, n (\%) & $7(15.2)$ & $13(34.2)$ & 0.070 & $1(6.3)$ & $5(20.0)$ & 0.390 & $5(35.7)$ & $8(33.3)$ & 1.000 \\
\hline Moderate OSA, n (\%) & $25(54.4)$ & $19(50.0)$ & 0.820 & $14(87.5)$ & $11(36.7)$ & $<0.002$ & $9(64.3)$ & $10(41.7$ & 0.313 \\
\hline Severe OSA, n (\%) & $14(30.4)$ & $6(15.8)$ & 0.132 & $1(6.3)$ & $13(43.3)$ & 0.016 & $0(0.0)$ & $6(25.0)$ & 0.067 \\
\hline Positional OSA, n (\%) & $25(73.5)$ & $23(76.7)$ & 1.000 & $13(92.9)$ & $12(60.0)$ & 0.050 & $10(83.3)$ & $13(72.2)$ & 0.669 \\
\hline
\end{tabular}

* (means + standard deviation)

AHI, apnea-hypopnea index; BMI, body mass index; Non-Sup, nonsupine; OSA, obstructive sleep apnea.

Table 3. Pretreatment, Custom oral appliance, and Apnea Guard apnea-hypopnea index values stratified by sex and tongue scallop

\begin{tabular}{|c|c|c|c|c|c|c|c|c|c|}
\hline & \multicolumn{3}{|c|}{ Mean \pm SD } & \multicolumn{3}{|c|}{$P$} & \multicolumn{3}{|c|}{ No. Patients } \\
\hline & PT & CA & AG & PT vs. CA & PT vs. AG & AG & PT & $\mathrm{CA}$ & $\mathrm{AG}$ \\
\hline \multicolumn{10}{|c|}{ Males: Nonscalloped } \\
\hline Overall & $22.1 \pm 6.1$ & $10.1 \pm 4.0$ & $7.2 \pm 2.5$ & $<0.00001$ & $<0.00001$ & ns & 16 & 16 & 16 \\
\hline Supine & $31.4+11.2$ & $14.8 \pm 8.6$ & $10.2 \pm 6.1$ & $<0.0005$ & $<0.00001$ & ns & 16 & 16 & 16 \\
\hline Non-supine & $8.5 \pm 4.2$ & $4.3 \pm 2.3$ & $5.4 \pm 3.8$ & $<0.005$ & $<0.05$ & ns & 14 & 14 & 14 \\
\hline \multicolumn{10}{|c|}{ Males: Scalloped } \\
\hline Overall & $31.2 \pm 16.8$ & $15.4 \pm 9.5$ & $9.9 \pm 5.7$ & $<0.00001$ & $<0.00001$ & $<0.01$ & 30 & 30 & 30 \\
\hline Supine & $42.7 \pm 22.3$ & $23.3 \pm 16.6$ & $14.3 \pm 8.7$ & $<0.0005$ & $<0.00001$ & ns & 26 & 26 & 26 \\
\hline Nonsupine & $19.1 \pm 13.6$ & $10.1 \pm 9.7$ & $7.1 \pm 5.7$ & $<0.01$ & $<0.0005$ & ns & 24 & 24 & 24 \\
\hline \multicolumn{10}{|c|}{ Females: Nonscalloped } \\
\hline Overall & $16.3 \pm 6.9$ & $8.1 \pm 4.8$ & $4.7 \pm 3.9$ & $<0.005$ & $<0.0005$ & ns & 14 & 14 & 14 \\
\hline Supine & $25.9 \pm 9.7$ & $13.9 \pm 11.6$ & $7.0 \pm 4.2$ & 0.01 & $<0.00001$ & ns & 14 & 14 & 12 \\
\hline Nonsupine & $5.0 \pm 5.4$ & $3.7 \pm 3.8$ & $4.1 \pm 4.8$ & ns & ns & ns & 12 & 12 & 13 \\
\hline \multicolumn{10}{|c|}{ Females: Scalloped } \\
\hline Overall & $22.9 \pm 15.2$ & $12.5 \pm 11.8$ & $8.7 \pm 7.6$ & $<0.01$ & $<0.00001$ & ns & 24 & 24 & 24 \\
\hline Supine & $27.0 \pm 16.0$ & $13.5 \pm 12.0$ & $9.3 \pm 7.8$ & $<0.005$ & $<0.00001$ & ns & 23 & 24 & 24 \\
\hline Nonsupine & $11.6 \pm 9.2$ & $9.8 \pm 10.2$ & $8.4 \pm 8.2$ & ns & $\mathrm{ns}$ & ns & 19 & 18 & 20 \\
\hline
\end{tabular}

AG, Apnea Guard; AHI, apnea-hypopnea index; CA, custom oral appliance; PT, pretreatment; SD, standard deviation.

reducing sleep-disordered breathing. Compared to the CA, the AG protocol delivered significantly greater reductions in AHI values, increased the percentage of patients who achieved an $\mathrm{AHI}<10$ events/h by $30 \%$, and those who achieved $\geq 50 \%$ AHI reduction by $25 \%$.

Previously, tongue size was associated with increased AHI severity, and tongue scallop was associated with increased intermittent hypoxemia attributed to sleepdisordered breathing. ${ }^{18.30,31}$ In this cohort of patients intolerant of PAP, tongue scallop was associated with increased PT nonsupine AHI values in both males and females, increased BMI in females, and decreased incident of POSA in males. The clinically accessible estimate of tongue size used in this study was predictive of those who achieving $\geq 50 \%$ AHI reduction, particularly in males. When the AG VDO was selectively increased based on sex and tongue scallop, BMI was predictive of therapeutic response across the three outcome criteria. It can be speculated that the association between obesity and OA outcomes was not observed with the CA because of variability introduced by the selection of VDO based only on sex.

Relatively small increases in VDO based on sex and tongue size potentially served two purposes. First, VDO advanced the jaw forward slightly and created greater room in the oral cavity to accommodate scalloped tongues with the OA in situ. Expansion of the oral cavity potentially reduced the need for excessive mandibular advancement to accommodate these large tongues, a condition that can lead to temporomandibular joint disorders, tooth pain, or tooth movement. ${ }^{29,32-35}$ Second, VDO and mandibular advancement may have combined to activate the genioglossus muscle and improve the posture of the hyoid bone, thereby increasing the patency of the pharyngeal 
Table 4. Logistic regression results for three posttreatment outcome criteria stratified across and by sex

\begin{tabular}{|c|c|c|c|c|c|c|c|c|c|}
\hline & \multicolumn{3}{|c|}{ Across sexes and OAs $(n=168)$} & \multicolumn{3}{|c|}{ Males - both OAs $(n=92)$} & \multicolumn{3}{|c|}{ Females - both OAs $(n=76)$} \\
\hline Variable & $\begin{array}{l}\text { Odds } \\
\text { Ratio }\end{array}$ & $(95 \% \mathrm{CI})$ & $P$ & $\begin{array}{l}\text { Odds } \\
\text { Ratio }\end{array}$ & $(95 \% \mathrm{CI})$ & $P$ & $\begin{array}{l}\text { Odds } \\
\text { Ratio }\end{array}$ & $(95 \% \mathrm{CI})$ & $P$ \\
\hline \multicolumn{10}{|c|}{ Predictors of Outcome Criteria: $\mathrm{AHI}<10$ events $/ \mathrm{h}$} \\
\hline Sex & 4.14 & $(1.0-17.2)$ & 0.051 & -- & - & - & -- & -- & -- \\
\hline Tongue scallop & 0.71 & $(0.30-1.70)$ & 0.447 & 0.36 & $(0.11-1.15)$ & 0.084 & 1.60 & $(0.38-6.77)$ & 0.522 \\
\hline BMI & 0.94 & $(0.85-1.05)$ & 0.280 & 0.92 & $(0.76-1.11)$ & 0.395 & 0.94 & $(0.79-1.11)$ & 0.457 \\
\hline PT AHI & 0.88 & $(0.83-0.92)$ & $<0.001$ & 0.90 & $(0.85-0.96)$ & $<0.001$ & 0.81 & $(0.72-0.91)$ & $<0.001$ \\
\hline VDO & 1.83 & $(1.37-2.44)$ & $<0.001$ & 2.27 & $(1.46-3.54)$ & $<0.001$ & 1.66 & $(1.09-2.52)$ & 0.018 \\
\hline \multicolumn{10}{|c|}{ Predictors of Outcome Criteria: $>50 \%$ AHI Reduction } \\
\hline Sex & 0.46 & $(0.21-0.99)$ & 0.046 & -- & -- & -- & -- & -- & -- \\
\hline Tongue scallop & 0.46 & $(0.21-0.99)$ & 0.047 & 0.20 & $(0.06-0.69)$ & 0.011 & 0.82 & $(0.28-2.39)$ & 0.712 \\
\hline BMI & 0.92 & $(0.84-1.00)$ & 0.043 & 0.89 & $(0.74-1.07)$ & 0.223 & 0.92 & $(0.83-1.02)$ & 0.113 \\
\hline PT AHI & 1.05 & $(1.02-1.08)$ & 0.004 & 1.12 & $(1.06-1.20)$ & $<0.001$ & 1.00 & $(0.96-1.05)$ & 0.873 \\
\hline VDO & 1.34 & $(1.09-1.64)$ & 0.006 & 2.05 & $(1.34-3.14)$ & $<0.001$ & 1.13 & $(0.87-1.46)$ & 0.361 \\
\hline \multicolumn{10}{|c|}{ Predictors of Outcome Criteria: $>50 \%$ AHI Reduction plus AHI $<10$ events $/ h$} \\
\hline Sex & 2.78 & $(0.88-8.75)$ & 0.082 & -- & -- & -- & -- & -- & -- \\
\hline Tongue scallop & 0.55 & $(0.26-1.15)$ & 0.112 & 0.27 & $(0.09-0.82)$ & 0.021 & 0.97 & $(0.33-2.86)$ & 0.951 \\
\hline BMI & 0.91 & $(0.83-1.00)$ & 0.043 & 0.90 & $(0.76-1.08)$ & 0.253 & 0.89 & $(0.78-1.01)$ & 0.069 \\
\hline PT AHI & 0.96 & $(0.93-0.99)$ & 0.014 & 0.98 & $(0.93-1.02)$ & 0.248 & 0.94 & $(0.89-0.99)$ & 0.028 \\
\hline VDO & 1.36 & $(1.10-1.67)$ & 0.004 & 1.84 & $(1.28-2.65)$ & $<0.001$ & 1.14 & $(0.87-1.50)$ & 0.338 \\
\hline
\end{tabular}

AHI, apnea-hypopnea index; BMI, body mass index; CI, confidence interval; PT, pretreatment; VDO, vertical dimension of occlusion

Table 5. Logistic regression results for custom oral appliance and Apnea Guard and three posttreatment outcome criteria

\begin{tabular}{|c|c|c|c|c|c|c|}
\hline & \multicolumn{3}{|c|}{ CA - both sexes $(n=84)$} & \multicolumn{3}{|c|}{ AG - both sexes $(n=84)$} \\
\hline Variable & $\begin{array}{l}\text { Odds } \\
\text { Ratio }\end{array}$ & $(95 \% \mathrm{CI})$ & $P$ & $\begin{array}{l}\text { Odds } \\
\text { Ratio }\end{array}$ & $(95 \% \mathrm{CI})$ & $P$ \\
\hline \multicolumn{7}{|c|}{ Predictors of Outcome Criteria: $\mathbf{A H I}<10$ events/h } \\
\hline Sex & 1.30 & $(0.24-6.97)$ & 0.758 & 0.98 & $(0.94-1.03)$ & 0.428 \\
\hline Tongue scallop & 1.05 & $(0.34-3.26)$ & 0.934 & -- & -- & -- \\
\hline BMI & 0.97 & $(0.84-1.12)$ & 0.682 & 0.80 & $(0.67-0.96)$ & $\mathbf{0 . 0 1 5}$ \\
\hline PT AHI & 0.90 & $(0.84-0.96)$ & $<0.002$ & 0.95 & $(0.91-1.00)$ & 0.045 \\
\hline VDO & -- & -- & -- & 1.12 & $(0.50-2.48)$ & 0.782 \\
\hline \multicolumn{7}{|c|}{ Predictors of Outcome Criteria: $\geq \mathbf{5 0 \%}$ AHI Reduction } \\
\hline Sex & 1.20 & $(0.29-4.89)$ & 0.804 & 0.05 & $(0.00-2.33)$ & 0.128 \\
\hline Tongue scallop & 1.04 & $(0.38-2.81)$ & 0.943 & -- & -- & -- \\
\hline BMI & 0.97 & $(0.87-1.09)$ & 0.634 & 0.83 & $(0.70-0.97)$ & 0.020 \\
\hline PT AHI & 1.04 & $(1.00-1.08)$ & $\mathbf{0 . 0 5 3}$ & 1.07 & $(1.01-1.14)$ & 0.026 \\
\hline VDO & -- & -- & -- & 0.19 & $(0.04-0.84)$ & $\mathbf{0 . 0 2 9}$ \\
\hline \multicolumn{7}{|c|}{ Predictors of Outcome Criteria: $\geq 50 \%$ AHI Reduction plus AHI $<10$ events/h } \\
\hline Sex & 1.93 & $(0.41-9.00)$ & 0.405 & 0.34 & $(0.04-2.94)$ & 0.330 \\
\hline Tongue scallop & 1.42 & $(0.48-4.22)$ & 0.526 & -- & -- & -- \\
\hline BMI & 0.96 & $(0.85-1.09)$ & 0.521 & 0.80 & $(0.68-0.96)$ & 0.014 \\
\hline PT AHI & 0.97 & $(0.92-1.01)$ & 0.137 & 0.95 & $(0.91-1.00)$ & 0.045 \\
\hline VDO & -- & -- & -- & 0.44 & $(0.20-0.96)$ & $\mathbf{0 . 0 3 9}$ \\
\hline
\end{tabular}

AHI, apnea-hypopnea index; BMI, body mass index; CA, custom oral appliance; Cl, confidence interval; PT, pretreatment; VDO, vertical dimension of occlusion.

airway during sleep. ${ }^{36,37}$ The VDO incorporated into the $\mathrm{AG}$ (i.e., $5.5,6.5$, or $8 \mathrm{~mm}$ ) was relatively similar to the 5 $\mathrm{mm}$ of VDO used by Marklund et al. ${ }^{15}$ and the $6 \mathrm{~mm}$ used by Aarab et al., ${ }^{32}$ in contrast with the $14 \mathrm{~mm}$ studied by Pitsis et al., ${ }^{38}$ approximately 7 to $20 \mathrm{~mm}$ investigated by Vroegop et al..$^{39}$ and 10 to $12 \mathrm{~mm}$ used by Rose et al. ${ }^{33} \mathrm{In}$ males, the AG VDO was 2.5 to $4.0 \mathrm{~mm}$ larger than the VDO used in studies with the SomnoDent ${ }^{\mathrm{TM}}$ MAS (SomnoMed, Sydney, Australia) $)^{7,13,16,40}$ and $\mathrm{Narval}^{\mathrm{TM}}$ (ResMed, San Diego, CA). ${ }^{14}$

Differences between the CA and AG outcomes were most apparent in females with nonscalloped tongues and 
males with scalloped tongues (i.e., CA had $\sim 3 \mathrm{~mm}$ less VDO). It can be speculated that in these females, the VDO prescribed for a CA (e.g., 2 to $3 \mathrm{~mm}$ ) was insufficient to effectively counteract airway collapsibility in the supine position. Because males tend to have longer airways, which are more prone to collapse, the additional VDO may have reduced adipose-related pharyngeal narrowing or collapse in those with scalloped tongues. ${ }^{20,31}$ In females with scalloped tongues, the CA and the AG outcomes were most similar (compared to the other subgroups) despite a 4-mm difference in VDO. Further investigations are needed to determine whether the relatively shorter female airway, in combination with greater body mass, more severe OSA, and elevated nonsupine AHI values, constitutes a phenotype that responds less favorably to OA therapy.

This study has a number of limitations. First, it could be argued that differences in outcomes purportedly explained by VDO may have resulted from an interaction between VDO and other OA functional settings. Following a conventional protocol, the $\mathrm{CA}$ was delivered at $70 \%$ advancement from the distance between maximum retrusion and maximum protrusion based on a George Gauge, with further adjustments made by the dentist. The AG protocol called for use of the "target" setting, determined with the $\mathrm{OA}$ in situ at $70 \%$ of the range from neutral centric occlusion to maximum protrusion. ${ }^{28} \mathrm{CA}$ vertical mouth opening was managed according to a conventional protocol, albeit in a relatively uncontrolled manner as compared to the AG. ${ }^{40}$ As a counterargument, when protrusion and vertical mouth opening were controlled, VDO was predictive in the AG logistic regression for the two outcome criteria requiring $\geq 50 \%$ AHI reductions.

Second, differences between the CA and AG were only assessed in patients who exhibited a response to the AG. Of the patients who failed to respond to the AG, $40 \%$ had a pretreatment $\mathrm{AHI}<20$ events $/ \mathrm{h}$, which required $\geq 30 \%$ AHI reduction, and $60 \%$ had a pretreatment $\mathrm{AHI} \geq 20$ events $/ \mathrm{h}$, which required $\geq 40 \%$ AHI reduction. It is unlikely that a sufficient portion of the AG nonresponders would have exhibited a superior CA treatment response sufficient to negate the primary finding (i.e., the AG protocol was superior).

Third, the sample sizes, when stratified by sex and tongue scallop, were relatively small. The results however, remained robust after the Bonferroni corrections. At minimum, these findings suggest interactions between sex, tongue scallop, and VDO that should be explored in an effort to improve OA outcomes.

Fourth, several CA design types were used in this study. This heterogeneity may have introduced variability which affected the capability to systematically determine why the AG provided superior outcomes relative to the CA. Conversely, OA design type was not predictive of outcomes in the CA logistic regression, and this study's results reflect what might be expected in a typical dental sleep medicine practice.

In conclusion, the outcomes achieved with the $\mathrm{AG}$ protocol relative to a conventional CA protocol may be explained by selection of OA settings that address different phenotypic characteristics. Preliminary results from a follow-up study suggest equivalent outcomes can be achieved when the CA is articulated to the VDO and target protrusion of the AG. In this follow-up study, the AG functional settings will be digitally scanned and transferred to the $\mathrm{CA}$ in an effort to improve OA fit during fabrication and reduce chair time upon delivery. ${ }^{41}$ Although limited to a maximum of 30 nights of use, the $A G$ provided sufficient time to objectively assess OAT response, and it delivered immediate therapeutic benefit while the patient waited for the CA to be fabricated.

\section{ABBREVIATIONS}

\section{AG Apnea Guard}

AHI apnea-hypopnea index

BMI body mass index

CA Custom appliance

HSAT home sleep apnea test

OA oral appliance

OAT oral appliance therapy

OSA obstructive sleep apnea

PAP positive airway pressure

POSA positional obstructive sleep apnea

PT pretreatment

VDO vertical dimension of occlusion

\section{ACKNOWLEDGMENTS}

The authors wish to thank Samuel Clark MD for his assistance in interpreting the HSAT studies and William Hevener, RPSGT for his assistance in identifying and referring patients for this study.

\section{REFERENCES}

1. Epstein LJ, Kristo D, Strollo PJ Jr, et al. Clinical guidelines for the evaluation, management, and long-term care of obstructive sleep apnea in adults. J Clin Sleep Med. 2009; 5(3): 263-276.

2. Ramar K, Dort LC, Katz SG, et al. Clinical practice guidelines for the treatment of obstructive sleep apnea and snoring with oral appliance therapy: an update for 2015. J Clin Sleep Med. 2015; 11(7): 773-827.

3. Sleep apnea oral appliances market by product (mandibular advancement devices (MAD) \& tongue-retaining devices (TRD)), Type (physician prescribed/customized oral appliances \& online OTC oral appliances) - global forecasts to 2023. Markets and Markets https://www.marketsandmarkets.com/Market-Reports/sleep-apneaoral-appliances-market-105423877.html

4. Sleep apnea devices market size, share and trends analysis report by product $\{$ therapeutic (PAP, oral, nasal, chin), diagnostic (respiratory polygraphs, actigraph, polysomnography, pulse oximeters)] by region, 2019-2025. https://www.grandviewresearch.com/industryanalysis/sleep-apnea-devices-market 
5. Exploring the economic benefits of OSA diagnosis and treatment. Commissioned by the American Academy of Sleep Medicine. https://aasm.org/advocacy/initiatives/economic-impact-obstructivesleep-apnea/

6. Chen H, Eckert DJ, van der Stelt PF, et al. Phenotypes of responders to mandibular advancement device therapy in obstructive sleep apnea patients: a systematic review and meta-analysis. Sleep Med Rev. 2020; epub DOI: 10.1016/j.smrv.2019.101229.

7. Phillips CL, Grunstein RR, Darendeliler MA, et al. Health outcomes of continuous positive airway pressure versus oral appliance therapy for obstructive sleep apnea: a randomized controlled trial. Am J Respir Crit Care Med. 2013; 187(8):879-887.

8. de Vries GE, Hoekema A, Vermeulen KM, et al. Clinical- and costeffectiveness of a mandibular advancement device versus continuous positive airway pressure in moderate obstructive sleep apnea. J Clin Sleep Med. 2019; 15(10):1477-1485.

9. Rapoport DM. Moving beyond empiric trial to using combined physiology and anatomy to predict success of oral appliances in obstructive sleep apnoea. J Physiol. 2019; 597(22):5321.

10. Chung JW, Enciso R, Levendowski DJ, et al. Treatment outcomes of mandibular advancement devices in positional and nonpositional OSA patients. Oral Surg Oral Med Oral Pathol Oral Radiol Endod. 2010; 109(5):724-731.

11. Remmers J, Charkhandeh S, Grosse J, et al. Remotely controlled mandibular protrusion during sleep predicts therapeutic success with oral appliances in patients with obstructive sleep apnea. Sleep. 2013; 36(10):1517-1525.

12. Remmers JE, Topor Z, Grosse J, et al. A feedback-controlled mandibular positioner identifies individuals with sleep apnea who will respond to oral appliance therapy. J Clin Sleep Med. 2017; 13(7):871880 .

13. Chen H, Aarab G, Lobbezoo F, et al. Differences in threedimensional craniofacial anatomy between responders and nonresponders to mandibular advancement splint treatment in obstructive sleep apnoea patients. Eur J Orthod. 2019; 41(3):308-315.

14. Vecchierini MF, Attali V, Collet JM, et al. Sex differences in mandibular repositioning device therapy effectiveness in patients with obstructive sleep apnea syndrome. Sleep Breath. 2019; 23:837-848.

15. Marklund M, Persson M, Franklin KA. Treatment success with a mandibular advancement device is related to supine-dependent sleep apnea. Chest. 1998; 114(6):1630-1635.

16. Sutherland K, Takaya H, Qian J, et al. Oral appliance treatment response and polysomnographic phenotypes of obstruct sleep apnea. J Clin Sleep Med. 2015; 11(8):861-868.

17. Bamagoos AA, Cistulli PA, Sutherland K, et al. Polysomnographic endotyping to select patients with obstructive sleep apnea for oral appliances. Ann Am Thorac Soc. 2019; 16(11):1422-1431.

18. Kim AM, Keenan BT, Jackson N, et al. Tongue fat and its relationship to obstructive sleep apnea. Sleep. 2014; 37(10):16391648.

19. Genta PR, Schorr FS, Eckert DJ, et al. Upper airway collapsibility is associated with obesity and hyoid position. Sleep. 2014; 37(10):16731678.

20. Malhotra A, Huang Y, Fogel RB, et al. The male predisposition to pharyngeal collapse: importance of airway length. Am J Respir Crit Care Med. 2002; 166(10):1388-1395.

21. Sutherland K, Chapman JL, Cayanan EA, et al. Parsing the craniofacial phenotype: effect of weight change in an obstructive sleep apnoea population. Sleep Breath. 2019; 23(4):1291-1298.

22. Sutherland K, Lee RWW, Chan TO, et al. Craniofacial phenotyping in Chinese and Caucasian patients in sleep apnea: influence of ethnicity and sex. J Clin Sleep Med. 2018; 14(7):1143-1151.

23. Shen HL, Wen YW, Chen NH, et al. Craniofacial morphologic predictors of oral appliance outcomes in patients with obstructive sleep apnea. J Am Dent Assoc. 2012; 143(11):1209-2017.
24. Marques M, Genta PR, Azarbarzin A, et al. Structure and severity of pharyngeal obstruction determine oral appliance efficacy in sleep apnoea. J Physiol. 2019; 597(22):5399-5410.

25. Sutherland K, Ngiam J, Cistulli PA. Performance of remotely controlled mandibular protrusion sleep studies for prediction of oral appliance treatment response. J Clin Sleep Med. 2017; 13(3):411417.

26. Levendowski DJ, Oksenberg A, Vicini C, et al. A systematic comparison of factors that could impact treatment recommendations for patients with Positional Obstructive Sleep Apnea (POSA). Sleep Med. 2018; 50:145-151.

27. Apnea Guard Theragnostic Protocol, Dec 2019. https://advancedbrainmonitoring.box.com/s/f8pqk3iind6b4zxnvn04

28. Levendowski D, Morgan T, Westbrook P. Initial evaluation of a titration appliance for temporary treatment of obstructive sleep apnea. J Sleep Disord Ther. 2012; 1(1):101.

29. de Ruiter MHT, Aarab G, de Vries N, et al. A stepwise titration protocol for oral appliance therapy in positional obstructive sleep apnea patients: proof of concept. Sleep Breath. 2020; 24(3):12291236.

30. Wang SH, Keenan BT, Wiemken A, et al. Effect of weight loss on upper airway anatomy and the apnea hypopnea index; the importance of tongue fat. Am J Respir Crit Care Med. 2020; 201(6):718-727.

31. Tomooka K, Tanigawa T, Sakurai S, et al. Scalloped tongue is associated with nocturnal intermittent hypoxia among communitydwelling Japanese: The Toon Health Study. J Oral Rehabil. 2017; 44(8):602-609.

32. Aarab G, Lobbezoo F, Hamburger HL, et al. Effects of an oral appliance with different mandibular protrusion positions at a constant vertical dimension on obstructive sleep apnea. Clin Oral Investig. 2010; 14(3):339-345.

33. Rose EC, Staats R, Virchow C, et al. A comparative study of two mandibular advancement appliances for the treatment of obstructive sleep apnoea. Eur J Orthod. 2002; 24(2):191-198.

34. Minagi HO, Okuno K, Nohara K, et al. Predictors of side effects with long-term oral appliance therapy for obstructive sleep apnea. J Clin Sleep Med. 2018; 14(1): 119-125.

35. Sakamoto Y, Furuhashi A, Komori E, et al. The most effective amount of forward movement for oral appliances for obstructive sleep apnea: A systematic review. Int J Environ Res Public Health. 2019; $16: 3248 ; 1-11$.

36. Long J, Ogawa T, Ito T, et al. Effect of bite opening and mandibular protrusion on genioglossus muscle activity in healthy adults with oral appliance. Odontology. 2018; 106(1):90-95.

37. Hiyama S. Tsuiki S, Ono T, et al. Effects of mandibular advancement on supine airway size in normal subjects during sleep. Sleep. 2003; 26(4): 440-445.

38. Pitsis AJ, Darendeliler MA, Gotsopoulos H, et al. Effect of vertical dimension on efficacy of oral appliance therapy in obstructive sleep apnea. Am J Respir Crit Care Med. 2002; 166(6):860-864.

39. Vroegop AVMT, Vanderveken OM, Van de Heyning PH, et al. Effects of vertical opening on pharyngeal dimensions in patients with obstructive sleep apnoea. Sleep Med. 2012; 13(3):314-316.

40. Milano F, Mutinelli S, Sutherland K, et al. Influence of vertical mouth opening on oral appliance treatment outcome in positional obstructive sleep apnea. J Dent Sleep Med. 2018; 5(1):17-23.

41. Charkhandeh S, Kuhns D, Kim S. A fully digital workflow and device manufacturing for mandibular repositioning devices for the treatment of obstructive sleep apnea: A feasibility study. J Dent Sleep Med. 2018; 4(4):97-102. 


\section{SUBMISSION \& CORRESPONDENCE INFORMATION}

Submitted in final revised form November 4, 2020 Accepted for publication January 8, 2021

Address correspondence to: Daniel Levendowski MBA, Advanced Brain Monitoring, Inc., 2237 Faraday Avenue, Carlsbad, CA, USA. Email: Dan@b-alert.com

\section{DISCLOSURE STATEMENT}

Levendowski and Morgan invented the Apnea Guard; however, neither receive financial benefit unless the intellectual property were to be sold to a third party. Sall is the Medical Director of Prosomnus Sleep Technologies; he had no influence on the custom OAs selected and fitted in this study. The other authors report no conflicts of interest. 